Research Article

\title{
Adaptive Backstepping Controller Design for the Anti-Synchronization of Identical WINDMI Chaotic Systems with Unknown Parameters and its SPICE Implementation
}

\author{
S. Vaidyanathan ${ }^{1}$, Ch. K. Volos ${ }^{*}$, , K. Rajagopal ${ }^{3}$, I. M. Kyprianidis ${ }^{2}$ and I. N. Stouboulos ${ }^{2}$ \\ ${ }^{I}$ Research and Development Centre, Vel Tech University, Avadi, Chennai-600062, Tamil Nadu, India. \\ ${ }^{2}$ Department of Physics, Aristotle University of Thessaloniki, Thessaloniki, GR-54124, Greece. \\ ${ }^{3}$ Department of Communication Engineering, Defence Engineering College, DebreZeit, Ethiopia.
}

Received 2 September 2014; Revised 24 October 2014; Accepted 5 November 2014

\begin{abstract}
This paper derives new results for the adaptive backstepping controller design for the anti-synchronization of identical WINDMI systems (Wind-Magnetosphere-Ionosphere models) with unknown parameters and also details the SPICE implementation of the proposed adaptive backstepping controller. In the anti-synchronization of chaotic systems, the sum of the outputs of master and slave systems is made to converge asymptotically to zero with time. The adaptive controller design for the anti-synchronization of identical WINDMI systems with unknown parameters has been established by applying Lyapunov stability theory. MATLAB simulations have been shown for the illustration of the adaptive anti-synchronizing backstepping controller for identical WINDMI chaotic systems. Finally, the proposed controller has been implemented using SPICE and circuit simulation results have been detailed.
\end{abstract}

Keywords: Chaos, chaotic systems, anti-synchronization, WINDMI system, stability, SPICE simulation.

\section{Introduction}

Chaotic systems can be defined as nonlinear dynamical systems which are sensitive to initial conditions, topologically mixing and also with dense periodic orbits [1]. The sensitivity to initial conditions of a chaotic system is indicated by a positive Lyapunov exponent. A dissipative chaotic system is characterized by the condition that the sum of the Lyapunov exponents of the chaotic system is negative.

A great breakthrough in chaos theory occurred when Lorenz discovered a 3-D chaotic system, while he was studying weather patterns [2]. There are many paradigms of 3-D chaotic systems in the literature, such as Rössler system [3], Rabinovich system [4], ACT system [5], Sprott systems [6], Chen system [7], Lü system [8], Shaw system [9], Feeny system [10], Shimizu system [11], Liu-Chen system [12], Cai system [13], Tigan system [14], Colpitt's oscillator [15], WINDMI system [16], Zhou system [17], etc.

Recently, many 3-D chaotic systems have been discovered such as Li system [18], Elhadj system [19], Pan system [20], Sundarapandian system [21], Yu-Wang system [22], Sundarapandian-Pehlivan system [23], Zhu system [24], Vaidyanathan systems [25-31], VaidyanathanMadhavan system [32], Pehlivan-Moroz-Vaidyanathan system [33], Jafari system [34], Pham system [35], etc.

\footnotetext{
* E-mail address: volos@physics.auth.gr ISSN: 1791-2377 @ 2015 Kavala Institute of Technology. All rights reserved.
}

The study of chaos theory in the last few decades had a big impact on the foundations of Science and Engineering and has found several engineering applications.

Some important applications of chaos theory can be cited as oscillators [36-38], lasers [39,40], robotics [41-43], chemical reactors $[44,45]$, biology $[46,47]$, ecology $[48,49]$, neural networks [50-52], secure communications [53-56], cryptosystems [57-61], economics [62-64], etc.

The phenomenon of anti-synchronization of chaotic systems can be stated as follows. If a particular chaotic system is called the master system and another chaotic system is called the slave system, then the idea of antisynchronization is to use the output of the master system to control the output of the slave system so that the outputs of the master and slave systems have the same amplitude but opposite signs asymptotically. Thus, in the antisynchronization of chaotic systems, the sums of the states of the master and slave systems are expected to converge to zero asymptotically with time. This is an important research problem with several applications in engineering [65].

In the chaos literature, many different methodologies have been proposed for the control of chaotic systems such as active control method [66-79], adaptive control method [70-76], backstepping control method [77-79], sliding mode control [80-81], etc.

In the chaos literature, many different methodologies have been also proposed for the synchronization and antisynchronization of chaotic systems such as PC method [82], active control [83-93], time-delayed feedback control 
[94,95], adaptive control [96-107], sampled-data feedback control [108-111], backstepping control [112-118], sliding mode control [119-124], etc.

In this research paper, we design an adaptive backstepping controller for the anti-synchronization of identical WINDMI chaotic systems with unknown parameters. WINDMI chaotic systems and WindMagnetosphere-Ionosphere models [16], which describe the energy flow through the solar wind-magnetosphereionosphere system.

We apply Lyapunov stability theory to establish the main result for the adaptive backstepping controller for the antisynchronization of identical WINDMI chaotic systems with unknown parameters. MATLAB simulations have been depicted to validate and illustrate the main results of this research work. Finally, we detail SPICE implementation of the adaptive backstepping controller proposed in this research work for the anti-synchronization of identical WINDMI chaotic systems with unknown parameters.

\section{Analysis on WINDMI Chaotic System}

WINDMI chaotic system is one of the paradigms of 3-D chaotic systems. It is described by the following normalized state equations:

$$
\left\{\begin{array}{l}
\frac{d x_{1}}{d t}=x_{2} \\
\frac{d x_{2}}{d t}=x_{3} \\
\frac{d x_{3}}{d t}=-a x_{3}-x_{2}+b-\exp \left(x_{1}\right)
\end{array}\right.
$$

where $x_{1}, x_{2}, x_{3}$ are the states and $a, b$ are positive parameters.

The WINDMI system (1) depicts a strange chaotic attractor when the parameter values are taken as:

$a=0.7$ and $b=2.5$

Also, we take the initial conditions as:

$x_{1}(0)=1.4, x_{2}(0)=0.8, x_{3}(0)=2.5$

The 3-D phase portrait of the WINDMI chaotic attractor is shown in Fig. 1.

The Lyapunov exponents of the WINDMI chaotic system are numerically obtained as:

$L_{1}=0.0845, L_{2}=0, L_{3}=-0.7870$

Thus, the maximal Lyapunov exponent (MLE) of the WINDMI chaotic system is given by $L_{1}=0.0845$.

The spectrum of the Lyapunov exponents of the WINDMI chaotic system (1) is depicted in Fig. 2.

The Lyapunov dimension of the WINDMI system is calculated as follows:

$D_{L}=2+\frac{L_{1}+L_{2}}{\left|L_{3}\right|}=2.1074$

which is fractional.

Since the sum of the Lyapunov exponents in (4) is negative, the WINDMI chaotic system is a dissipative chaotic system.

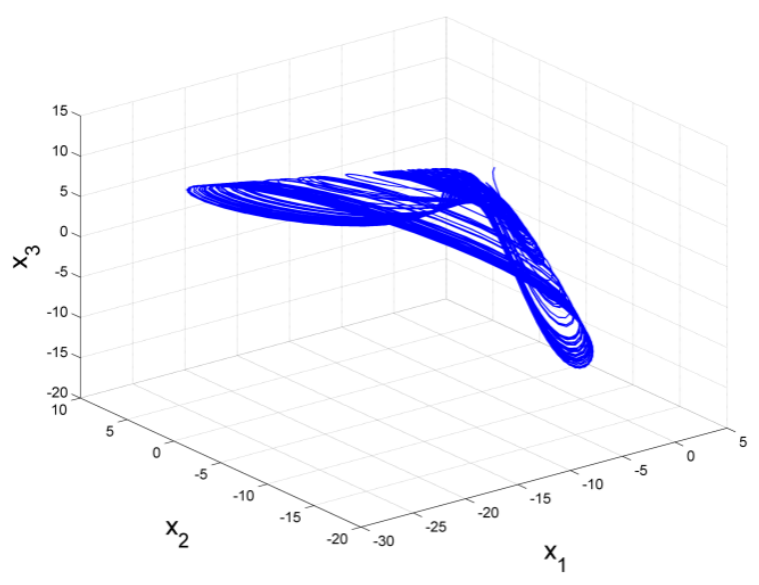

Fig. 1.The strange attractor of the WINDMI chaotic system.

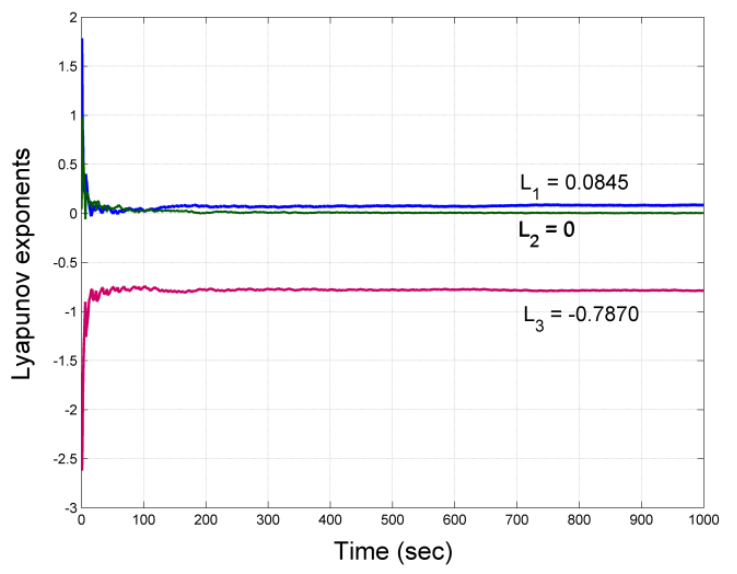

Fig. 2.The spectrum of the Lyapunov exponents of the WINDMI chaotic system.

\section{Adaptive Anti-Synchronization of WINDMI Chaotic Systems via Backstepping Control}

In this section, new results are derived for the antisynchronization of WINDMI chaotic systems with unknown parameters via adaptive backstepping control method.

As the master system, we consider the WINDMI system of Eq.(1), while as the slave system, the following controlled WINDMI system is considered.

$$
\left\{\begin{array}{l}
\frac{d y_{1}}{d t}=y_{2} \\
\frac{d y_{2}}{d t}=y_{3} \\
\frac{d y_{3}}{d t}=-a y_{3}-y_{2}+b-\exp \left(y_{1}\right)+u
\end{array}\right.
$$

In (6), the parameters $a$ and $b$ are unknown, and $u(t)$ is a feedback control to be determined using the states of (1) and (6), and estimates $A(t)$ and $B(t)$ of the unknown parameters $a$ and $b$, respectively.

The anti-synchronization error between the states of the WINDMI systems (1) and (6) is defined by

$\left\{\begin{array}{l}e_{1}(t)=y_{1}(t)+x_{1}(t) \\ e_{2}(t)=y_{2}(t)+x_{2}(t) \\ e_{3}(t)=y_{3}(t)+x_{3}(t)\end{array}\right.$ 
A simple calculation yields the error dynamics as:

$\left\{\begin{array}{l}\frac{d e_{1}}{d t}=e_{2} \\ \frac{d e_{2}}{d t}=e_{3} \\ \frac{d e_{3}}{d t}=-a e_{3}-e_{2}+2 b-\exp \left(y_{1}\right)-\exp \left(x_{1}\right)+u\end{array}\right.$

We define the parameter estimation errors as:

$\left\{\begin{array}{l}e_{a}(t)=a-A(t) \\ e_{b}(t)=b-B(t)\end{array}\right.$

In (10), $A(t)$ and $B(t)$ are estimates for the unknown parameters $a$ and $b$ respectively, which will be determined using adaptive control theory.

It follows from (9) that

$\left\{\begin{array}{l}\frac{d e_{a}}{d t}=-\frac{d A}{d t} \\ \frac{d e_{b}}{d t}=-\frac{d B}{d t}\end{array}\right.$

Next, we shall state and prove the main result of this section.

Theorem 1. The identical WINDMI chaotic systems (6) and (7) with unknown parameters $a$ and $b$ are globally and exponentially anti-synchronized by the adaptive backstepping control law

$$
\begin{aligned}
u(t)=-2 B(t) & -3 e_{1}-4 e_{2}-k z_{3} \\
& +(A(t)-3) e_{3}+\exp \left(y_{1}\right)+\exp \left(x_{1}\right)
\end{aligned}
$$

where $k>0$ is a gain constant, with

$z_{3}=2 e_{1}+2 e_{2}+e_{3}$

and the update law for the parameter estimates is given by

$\left\{\begin{array}{l}\frac{d A}{d t}=-e_{3} z_{3} \\ \frac{d B}{d t}=2 z_{3}\end{array}\right.$

Proof. We prove this result via backstepping control method and Lyapunov stability theory.

First, we define a quadratic Lyapunov function

$V_{1}\left(z_{1}\right)=\frac{1}{2} z_{1}^{2}$

where

$z_{1}=e_{1}$

Differentiating $V_{1}$ along the dynamics (8), we get

$\frac{d V_{1}}{d t}=e_{1} e_{2}=-z_{1}^{2}+z_{1}\left(e_{1}+e_{2}\right)$

Now, we define

$z_{2}=e_{1}+e_{2}$

Using (17), we can simplify (16) as: $\frac{d V_{1}}{d t}=-z_{1}^{2}+z_{1} z_{2}$

Next, we define a quadratic Lyapunov function

$V_{2}\left(z_{1}, z_{2}\right)=V_{1}\left(z_{1}\right)+\frac{1}{2} z_{2}^{2}=\frac{1}{2}\left(z_{1}^{2}+z_{2}^{2}\right)$

Differentiating $V_{2}$ along the dynamics (9), we obtain

$\frac{d V_{2}}{d t}=-z_{1}^{2}-z_{2}^{2}+z_{2}\left(2 e_{1}+2 e_{2}+e_{3}\right)$

Now, we define

$z_{3}=2 e_{1}+2 e_{2}+e_{3}$

Using (21), we can simplify (20) as:

$\frac{d V_{2}}{d t}=-z_{1}^{2}-z_{2}^{2}+z_{2} z_{3}$

Finally, we define a quadratic Lyapunov function

$V\left(\mathbf{z}, e_{a}, e_{b}\right)=V_{2}\left(z_{1}, z_{2}\right)+\frac{1}{2} z_{3}^{2}+\frac{1}{2} e_{a}^{2}+\frac{1}{2} e_{b}^{2}$

From (23), it is clear that $V$ is a positive definite function on $R^{5}$.

The time-derivative of $V$ is calculated as:

$\frac{d V}{d t}=-z_{1}^{2}-z_{2}^{2}-z_{3}^{2}+z_{3} S-e_{a} \frac{d A}{d t}-e_{b} \frac{d B}{d t}$

where

$S=z_{3}+z_{2}+\frac{d z_{3}}{d t}=z_{3}+z_{2}+2 \frac{d e_{1}}{d t}+2 \frac{d e_{2}}{d t}+\frac{d e_{3}}{d t}$

Simplifying the equation (25), we obtain

$$
\begin{gathered}
S=2 b+3 e_{1}+4 e_{2}+(3-a) e_{3}-\exp \left(y_{1}\right) \\
-\exp \left(x_{1}\right)+u
\end{gathered}
$$

Substituting the control law (11) into (26), we get

$S=2(b-B(t))-(a-A(t)) e_{3}-k z_{3}$

Using (9), we can simplify the equation (27) as:

$S=2 e_{b}-e_{a} e_{3}-k z_{3}$

Substituting the value of $S$ from (28) into (24), we obtain

$$
\begin{gathered}
\frac{d V}{d t}=-z_{1}^{2}-z_{2}^{2}-(1+k) z_{3}^{2}+e_{a}\left(-z_{3} e_{3}-\frac{d A}{d t}\right) \\
+e_{b}\left(2 z_{3}-\frac{d B}{d t}\right)
\end{gathered}
$$

Substituting the update law (13) into (29), we obtain

$$
\frac{d V}{d t}=-z_{1}^{2}-z_{2}^{2}-(1+k) z_{3}^{2}
$$

Thus, it is clear that $\frac{d V}{d t}$ is a negative semi-definite function on $R^{5}$.

From (30), it follows that the vector $\boldsymbol{z}(t)=\left(z_{1}(t), z_{2}(t)\right.$, $\left.z_{3}(t)\right)$ and the parameter estimation error $\left(e_{a}(t), e_{b}(t)\right)$, are globally bounded, i.e. 


$$
\left[z_{1}(t) \quad z_{2}(t) \quad z_{3}(t) \quad e_{a}(t) \quad e_{b}(t)\right] \in \boldsymbol{L}_{\infty}
$$

Also, it follows from (30) that

$\frac{d V}{d t} \leq-z_{1}^{2}-z_{2}^{2}-z_{3}^{2}=-\|\mathbf{z}\|^{2}$

That is,

$$
\|\mathbf{z}\|^{2} \leq-\frac{d V}{d t}
$$

Integrating the inequality (34) from 0 to $t$, we get

$$
\int_{0}^{t}\|\mathbf{z}(\tau)\|^{2} d \tau \leq V(0)-V(t)
$$

From (49), it follows that $\boldsymbol{z}(t) \in \boldsymbol{L}_{2}$, while from (8), it can be deduced that $\frac{d z}{d t} \in \boldsymbol{L}_{\infty}$.

Thus, using Barbalat's lemma [125], we can conclude that $\mathbf{z}(t) \rightarrow 0$ exponentially as $t \rightarrow \infty$ for all initial conditions $\boldsymbol{z}(0) \in \boldsymbol{R}^{3}$.

Hence, it is immediate that the anti-synchronization error $e(t) \rightarrow 0$ exponentially as $t \rightarrow \infty$ for all initial conditions $\boldsymbol{e}(0) \in \boldsymbol{R}^{3}$.

Thus, it follows that WINDMI chaotic systems (1) and (6) are globally and exponentially anti-synchronized for all initial conditions $x(0), y(0) \in \boldsymbol{R}^{3}$.

This completes the proof.

For numerical simulations, the classical fourth-order Runge-Kutta method with step size $h=10^{-8}$ is used to solve the system of differential equations (1), (6) and (13), when the adaptive control law (11) is applied.

The parameter values of the WINDMI chaotic systems (1) and (6) are taken as in the chaotic case, viz. $a=0.7$ and $b=2.5$. The positive gain constant $k$ is taken as $k=6$.

Furthermore, as initial conditions of the master system (1), we take $x_{1}(0)=3.8, x_{2}(0)=-4.5$ and $x_{3}(0)=6.2$.

As initial conditions of the slave system (7), we take $y_{1}(0)=-7.4, y_{2}(0)=-3.6$ and $y_{3}(0)=4.9$.

Also, as initial conditions of the estimates $A(t)$ and $B(t)$, we take $A(0)=5.1$ and $B(0)=6.4$.

In Figs. 3-5, the anti-synchronization of the states of the master system (1) and slave system (6) is depicted, when the adaptive control law (11) and parameter update law (13) are implemented. In Fig. 6, the time-history of the antisynchronization errors $e_{1}(t), e_{2}(t), e_{3}(t)$ is depicted.

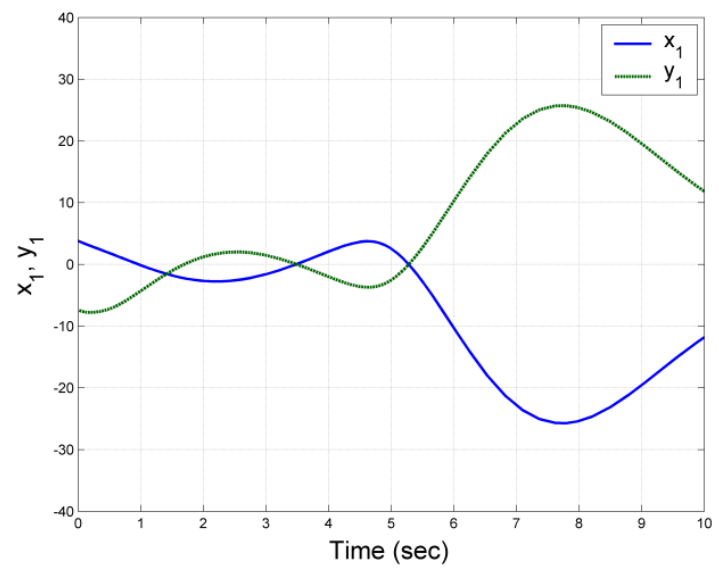

Fig. 3. Anti-Synchronization of the states $x_{1}(t)$ and $y_{1}(t)$.

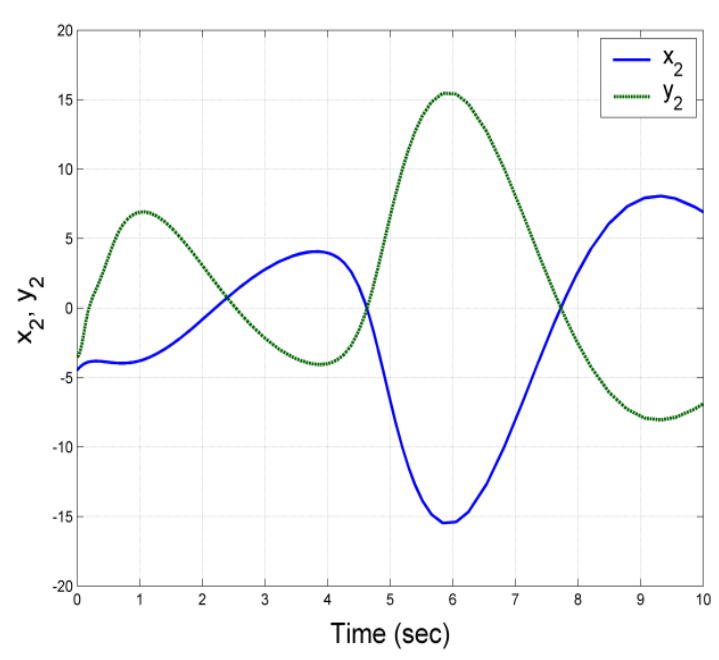

Fig. 4. Anti-Synchronization of the states $x_{2}(t)$ and $y_{2}(t)$.

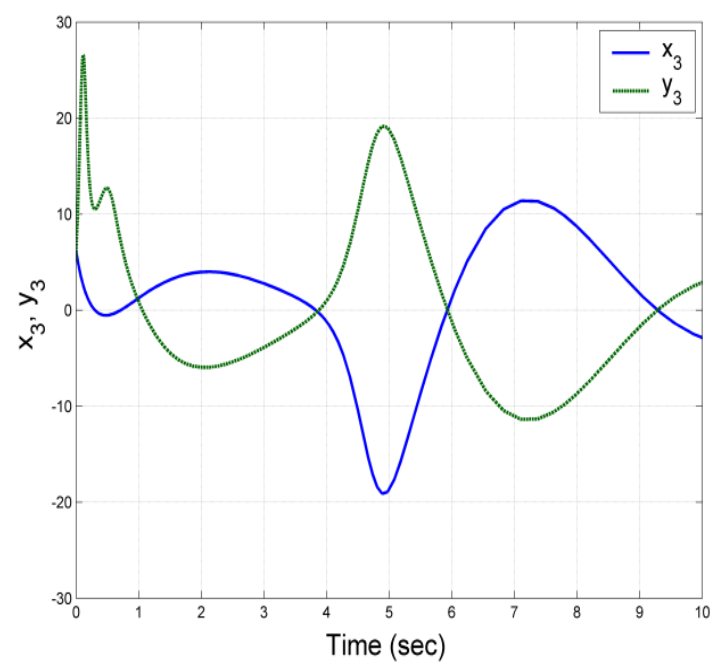

Fig. 5. Anti-Synchronization of the states $x_{3}(t)$ and $y_{3}(t)$.

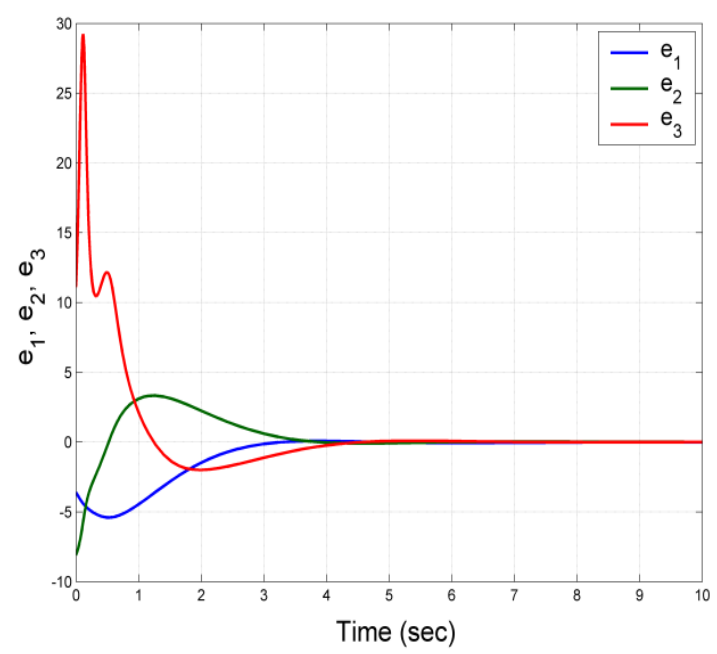

Fig. 6. Time-history of the anti-synchronization errors $\mathrm{e}_{1}(\mathrm{t}), \mathrm{e}_{2}(\mathrm{t}), \mathrm{e}_{3}(\mathrm{t})$. 


\section{Circuit Realization of the Adaptive Backstepping Controller Design for the Anti-Synchronization of WINDMI Chaotic Systems}

In this section, we design an electronic circuit modellingWINDMI system (1) and the controller (11) used for synchronization. The circuits in Figs. $7 \& 8$ has been designed following an approach based on operational amplifiers $[59,60,70]$ where the state variables $x_{1}, x_{2}$, and $x_{3}$ of the system (1) are associated with the voltages across the capacitors $C_{1}, C_{2}$, and $C_{3}$, respectively. In Figs. $7 \& 8$, there are five operational amplifiers, which are connected as integrators $\left(U_{1}, U_{2}, U_{3}, U_{4}\right.$ and $\left.U_{5}\right)$ and one as inverting amplifier $\left(U_{6}\right)$. The nonlinear equations for the electronic circuit are derived as follows:

$$
\left\{\begin{array}{l}
\frac{d x_{1}}{d t}=\frac{1}{R 1 C 1} x_{2} \\
\frac{d x_{2}}{d t}=\frac{1}{R 2 C 2} x_{3} \\
\frac{d x_{3}}{d t}=-\frac{1}{R 3 C 3} x_{3}-\frac{1}{R 4 C 3} x_{2}+\frac{1}{R 5 C 3}-\frac{1}{R 6 C 3} \exp \left(x_{1}\right)
\end{array}\right.
$$

where the values of components are chosen as: $R_{1}=R_{2}=R_{3}$ $=R_{5}=R_{7}=R_{8}=R_{9}=R_{10}=R_{11}=R_{12}=R_{13}=R_{14}=R_{15}=R_{16}$ $=R_{17}=R_{18}=R_{19}=R_{20}=1 \mathrm{k} \Omega, R_{4}=0.4 \mathrm{k} \Omega, R_{6}=1.428 \mathrm{k} \Omega$ and $C_{1}=C_{2}=C_{3}=C_{4}=C_{5}=1 \mathrm{mF}$. The power supplies of all active devices are $\pm 15 \mathrm{~V}_{\mathrm{DC}}$.

Figures $9,10 \& 11$ show the state portraits of $x_{1}$ versus $x_{2}, x_{2}$ versus $x_{3}$ and $x_{3}$ versus $x_{1}$, respectively.

\section{Conclusion}

In this work the adaptive backstepping controller design for the anti-synchronization of identical third-order chaotic systems with unknown parameters was studied. For this reason, an interesting system, such as the WindMagnetosphere-Ionosphere model (WINDMI) was chosen. The proposed controller design has been established by applying the Lyapunov stability theory. The simulation results, with MATLAB, confirmed the effectiveness of the adaptive anti-synchronization controller in the case of identical chaotic systems. Finally, the circuitries of the chosen dynamical system and the system's controller have been realized and the very satisfactory agreement of the SPICE results with those of MATLAB simulations has been observed.

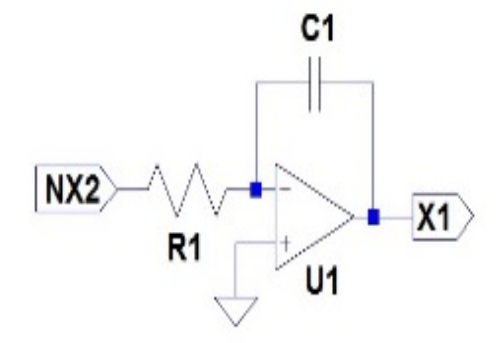

Fig.7.LTSpice schematic for master system.

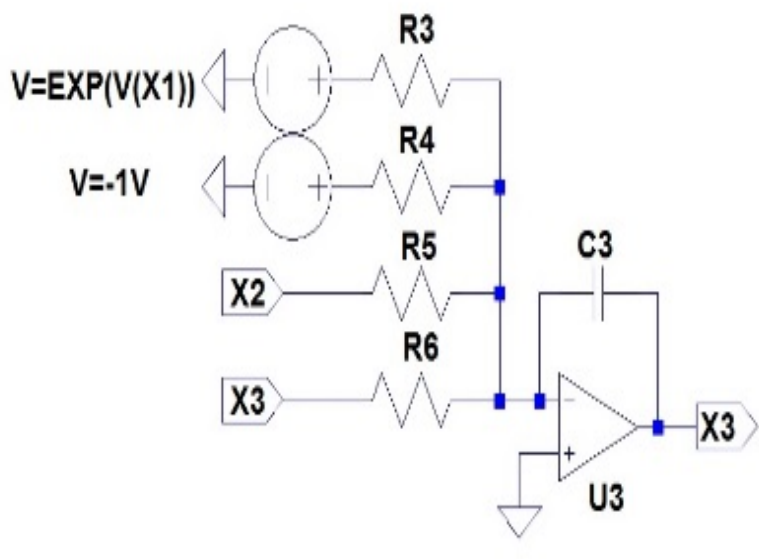



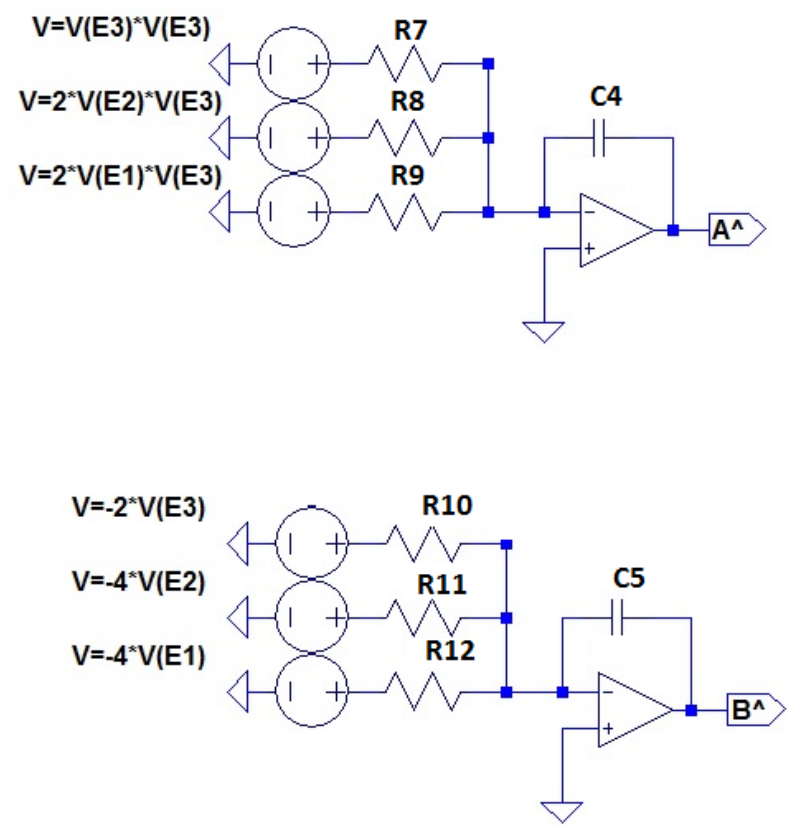

Fig.8. LTSpice schematic for controller.

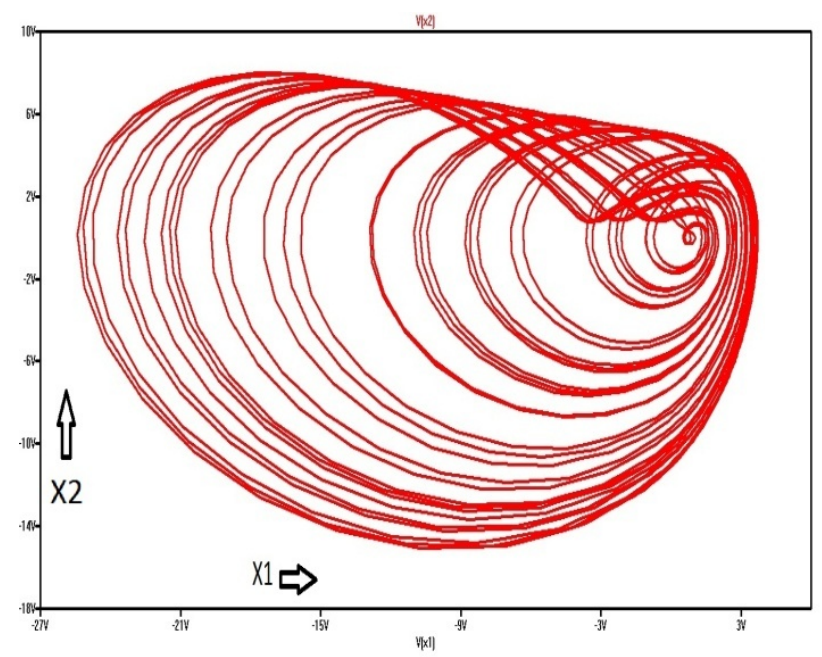

Fig.9. Phase portrait of $x_{1}$ vs. $x_{2}$

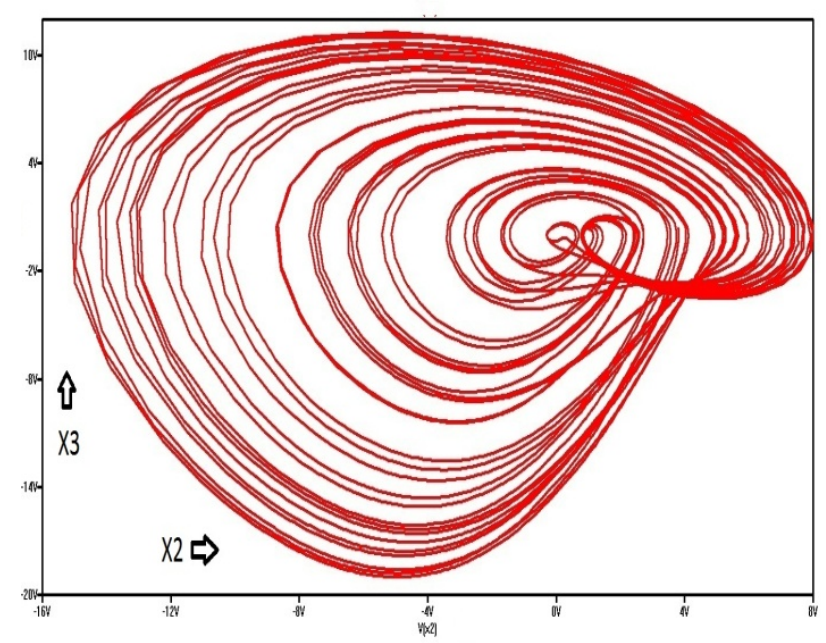

Fig.10.Phase portrait of $x_{2}$ vs. $x_{3}$.
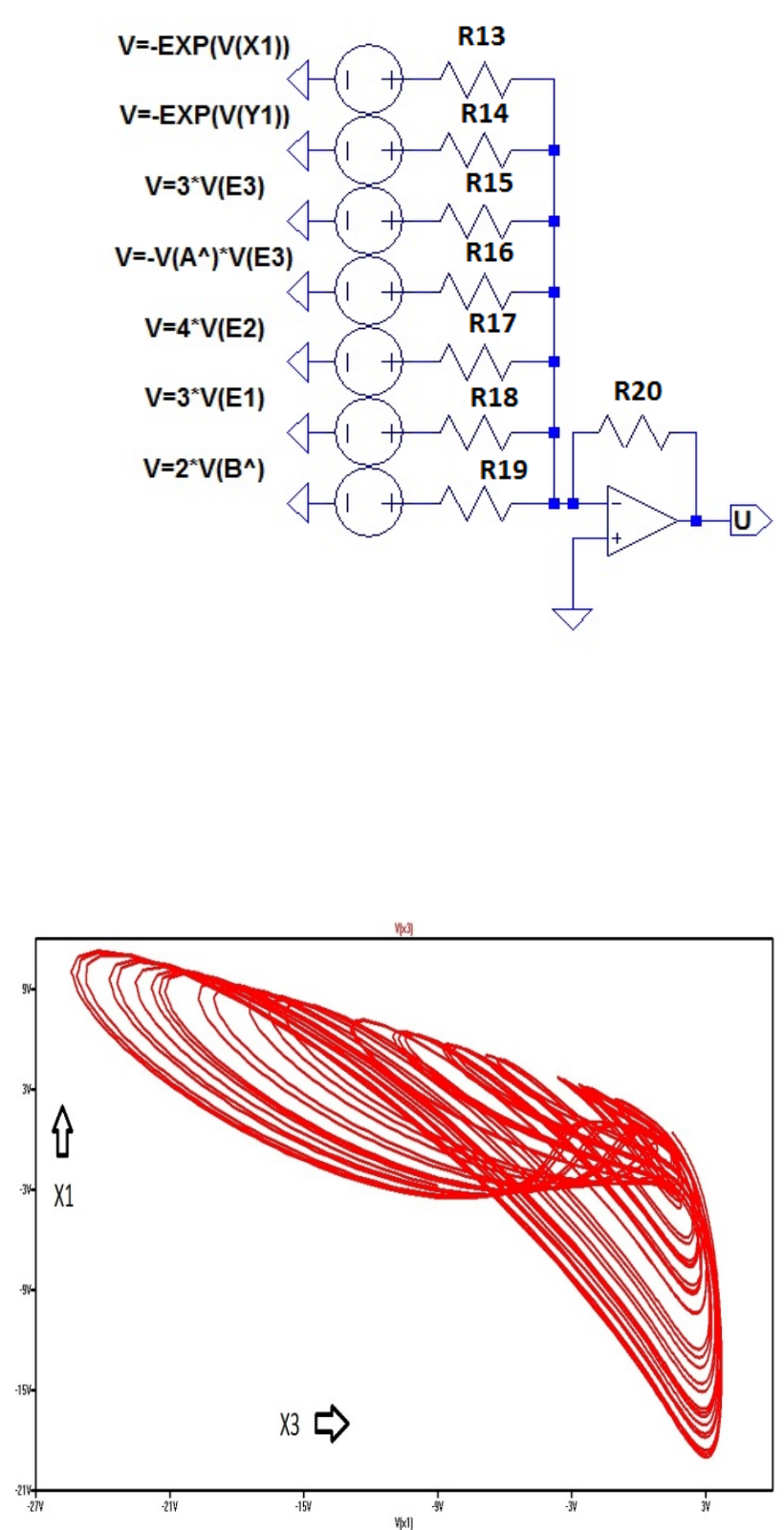

Fig.11. Phase portrait of $x_{3}$ vs. $x_{1}$. 


\section{References}

1. S.H. Strogatz, Nonlinear dynamics and chaos: With applications to physics, biology, chemistry, and engineering, Perseus Books, Massachusetts, US (1994)

2. E.N. Lorenz, Deterministic nonperiodic flow, Journal of the Atmospheric Sciences, vol. 20, pp. 130-141 (1963).

3. O.E. Rössler, An equation for continuous chaos, Physics Letters A, vol. 57, pp. 397-398 (1976)

4. M.I. Rabinovich and A.L. Fabrikant, Stochastic self-modulation of waves in nonequilibrium media, Sov. Phys. JETP, vol. 50, pp. 311-317, (1979)

5. A. Arneodo, P. Coullet, and C. Tresser, Possible new strange attractors with spiral structure, Communications in Mathematical Physics, vol. 79, pp. 573-579 (1981)

6. J.C. Sprott, Some simple chaotic flows, Physical Review E, vol. 50, pp. 647-650 (1994).

7. G. Chen and T. Ueta, Yet another chaotic oscillator, International Journal of Bifurcation and Chaos, vol. 9, pp. 14651466 (1999).

8. J. Lü and G. Chen, A new chaotic attractor coined, International Journal of Bifurcation and Chaos, vol. 12, pp. 659-661 (2002).

9. R. Shaw, Strange attractors, chaotic behaviour and information flow, Zeitschrift für Naturforschung, vol. 36, pp. 80-112 (1981).

10. B. Feeny and F.C. Moon, Chaos in a forced dry-friction oscillator: Experiments and numerical modeling, Journal of Sound and Vibration, vol. 170, pp. 303-323 (1994)

11. T. Shimizu and N. Moroika, On the bifurcation of a symmetric limit cycle to an asymmetric one in a simple model, Physics Letters A, vol. 76, pp. 201-204 (1980).

12. W. Liu and G. Chen, A new chaotic system and its generation, International Journal of Bifurcation and Chaos, vol. 13, pp. 261267 (2003).

13. G. Cai and Z. Tan, Chaos synchronization of a new chaotic system via nonlinear control, Journal of Uncertain Systems, vol. 1, pp. 235-240 (2007).

14. G. Tigan and D. Opris, Analysis of a 3D chaotic system, Chaos, Solitons and Fractals, vol. 36, pp. 1315-1319 (2008).

15. G.P. Kennedy, Chaos in the Colpitts oscillator, IEEE Transactions on Circuits and Systems-I, vol. 41, pp. 771-774 (1994).

16. J. Wang, D. Lu and L. Tian, Global synchronization for time delay of WINDMI system, Chaos, Solitons and Fractals, vol. 30, pp. 629-635 (2006)

17. W. Zhou, Y. Xu, H. Lu, and L. Pan, On dynamics analysis of a new chaotic attractor, Physics LettersA, vol. 372, pp. 5773-5777 (2008).

18. D. Li, A three-scroll chaotic attractor, Physics Letters A, vol. 372, pp. 387-393 (2008)

19. Z. Elhadj, Dynamical analysis of a 3-D chaotic system with only two quadratic nonlinearities, Journal of Systems Science and Complexity, vol. 21, pp. 67-75 (2008).

20. L. Pan, D. Xuand W. Zhou, Controlling a novel chaotic attractor using linear feedback, Journal of Information and Computing Science, vol. 5, pp. 117-124 (2010).

21. V. Sundarapandian, Analysis and anti-synchronization of a novel chaotic system via active and adaptive controllers, Journal of Engineering Science and Technology Review, vol. 6, pp. 45-52 (2013).

22. F. Yu, C. Wang, Q. Wan, and Y. Hu, Complete switched modified function projective synchronization of a five-term chaotic system with uncertain parameters and disturbances, Pramana, vol. 80, pp. 223-235 (2013).

23. V. Sundarapandian and I. Pehlivan, Analysis, control, synchronization and circuit design of a novel chaotic system, Mathematical and Computer Modelling, vol. 55, pp. 1904-1915 (2012).

24. C. Zhu, Y. Liu, and Y. Guo, Theoretical and numerical study of a new chaotic system, Intelligent Information Management, vol. 2, pp. 104-109 (2010)

25. S. Vaidyanathan, A new six-term 3-D chaotic system with an exponential nonlinearity, Far East Journal of Mathematical Sciences, vol. 79, pp. 135-143 (2013).

26. S. Vaidyanathan, Analysis and adaptive synchronization of two novel chaotic systems with hyperbolic sinusoidal and cosinusoidal nonlinearity and unknown parameters, Journal of
Engineering Science and Technology Review, vol. 6, pp. 53-65 (2013).

27. S. Vaidyanathan, A new eight-term 3-D polynomial chaotic system with three quadratic nonlinearities, Far East Journal of Mathematical Sciences, vol. 84, pp. 219-226 (2014).

28. S. Vaidyanathan, Analysis, control and synchronization of a sixterm novel chaotic system with three quadratic nonlinearities, International Journal of Modelling, Identification and Control, vol. 22, pp. 41-53 (2014).

29. S. Vaidyanathan, Analysis and adaptive synchronization of eight-term 3-D polynomial chaotic systems with three quadratic nonlinearities, European Physical Journal: Special Topics, vol. 223, pp. 1519-1529 (2014).

30. S. Vaidyanathan, C. Volos, V.T. Pham, K. Madhavan and B.A Idowu, Adaptive backstepping control, synchronization and circuit simulation of a 3-D novel jerk chaotic system with two hyperbolic sinusoidal nonlinearities, Archives of Control Sciences, vol. 24(3), pp. 257-285 (2014).

31. S. Vaidyanathan, Generalized projective synchronisation of novel 3-D chaotic systems with an exponential nonlinearity via active and adaptive control, International Journal of Modelling, Identification and Control, vol. 22 (3), pp. 207-217, 2014.

32. S. Vaidyanathan and K. Madhavan, Analysis, adaptive control and synchronization of a seven-term novel 3-D chaotic system, International Journal of Control Theory and Applications, vol. 6, pp. 121-137 (2013)

33. I. Pehlivan, I.M. Moroz, and S. Vaidyanathan, Analysis, synchronization and circuit design of a novel butterfly attractor, Journal of Sound and Vibration, vol. 333, pp. 5077-5096 (2014).

34. S. Jafari and J.C. Sprott, Simple chaotic flows with aline equilibrium, Chaos, Solitons and Fractals, vol. 57, pp. 79-84, 2013.

35. V.T. Pham, C. Volos, S. Jafari, Z. Weiand X. Wang, Constructing a novel no-equilibrium chaotic system, International Journal of Bifurcation and Chaos, vol. 24, 1450073 (2014).

36. G.H. Li, S.P. Zhou and K. Yang, Controlling chaos in Colpitts oscillator, Chaos, Solitons and Fractals, vol. 33, pp. 582-587 (2007).

37. J. Kengne, J.C. Chedjou, G. Kenne, and K. Kyamakya, Dynamical properties and chaos synchronization of improved Colpitts oscillators, Communications in Nonlinear Science and Numerical Simulation, vol. 17, pp. 2914-2923 (2012).

38. A. Sharma, V. Patidar, G. Purohit, and K.K. Sud, Effects on the bifurcation and chaos in forced Duffing oscillator due to nonlinear damping, Communications in Nonlinear Science and Numerical Simulation, vol. 17, pp. 2254-2269 (2012).

39. S. Donati and S.K. Hwang, Chaos and high-level dynamics in coupled lasers and their applications, Progress in Quantum Electronics, vol. 36, pp. 293-341 (2012).

40. N. Li, W. Pan, L. Yan, B. Luo, and X. Zou, Enhanced chaos synchronization and communication in cascade-coupled semiconductor ring lasers, Communications in Nonlinear Science and Numerical Simulation, vol. 19, pp. 1874-1883 (2014).

41. U. Nehmzow and K. Walker, Quantitative description of robotenvironment interaction using chaos theory, Robotics and Autonomous Systems, vol. 53, pp. 177-193 (2005).

42. Ch.K. Volos, I.M. Kyprianidis, and I.N. Stouboulos, A chaotic path planning generator for autonomous mobile robots, Robotics and Autonomous Systems, vol. 60, pp. 651-656, (2012).

43. Ch.K. Volos, I.M. Kyprianidis, and I.N. Stouboulos, Experimental investigation on coverage performance of a chaotic autonomous mobile robot, Robotics and Autonomous Systems, vol. 61(12), pp. 1314-1322(2013).

44. J.C. Roux, Chaos in experimental chemical systems: two examples, North-Holland Mathematics Studies, vol. 103, pp. 345-352 (1985).

45. Y.N. Li, L. Chen, Z.S. Cai, and X.Z. Zhao, Study on chaos synchronization in the Belousov-Zhabotinsky chemical system, Chaos, Solitons and Fractals, vol. 17, pp. 699-707 (2003).

46. M. Kyriazis, Applications of chaos theory to the molecular biology of aging, Experimental Gerontology, vol. 26, pp. 569572 (1991). 
47. G. Böhm, Protein folding and deterministic chaos: Limits of protein folding simulations and calculations, Chaos, Solitons and Fractals, vol. 1, pp. 375-382 (1991).

48. J.C. Sprott, J.A. Vano, J.C. Wildenberg, M.B. Anderson, and J.K. Noel, Coexistence and chaos in complex ecologies, Physics Letters A, vol. 335, pp. 207-212 (2005).

49. B. Sahoo and S. Poria, The chaos and control of a food chain model supplying additional food to top-predator, Chaos, Solitons and Fractals, vol. 58, pp. 52-64 (2014).

50. G. He, Z. Cao, P. Zhu, and H. Ogura, Controlling chaos in a chaotic neural network, Neural Networks, vol. 16, pp. 11951200 (2003).

51. E. Kaslik and S. Sivasundaram, Nonlinear dynamics and chaos in fractional-order neural networks, Neural Networks, vol. 32, pp. 245-256 (2012).

52. I.M. Kyprianidis and A.T. Makri, Complex dynamics of FitzHugh-Nagumo type neurons coupled with gap junction under external voltage stimulation, Journal of Engineering Science and Technology Review, vol. 6(4), pp. 104-114(2013).

53. K. Suzuki and Y. Imai, Decryption characteristics in message modulation type chaos secure communication system using optical fiber ring resonators, Optics Communications, vol. 259, pp. 88-93 (2006).

54. X.Y. Wang and Y.F. Gao, A switch-modulated method for chaos digital secure communication based on user-defined protocol, Communications in Nonlinear Science and Numerical Simulation, vol. 15, pp. 99-104 (2010).

55. O.I. Moskalenko, A.A. Koronovskii, and A.E. Hramov, Generalized synchronization of chaos for secure communication: Remarkable stability to noise, Physics Letters A, vol. 374, pp. 2925-2931 (2010)

56. A. Abdullah, Synchronization and secure communication of uncertain chaotic systems based on full-order and reduced-order output-affine observers, Applied Mathematics and Computation, vol. 219, pp. 10000-10011 (2013).

57. N. Smaoui and A. Kanso, Cryptography with chaos and shadowing, Chaos, Solitons and Fractals, vol. 42, pp. 2312-2321 (2009).

R. Rhouma and S. Belghith, Cryptanalysis of a chaos-based cryptosystem on DSP, Communications in Nonlinear Science and Numerical Simulation, vol. 16, pp. 876-884 (2011).

58. V. Sundarapandian and I. Pehlivan, Analysis, control, synchronizationand circuit design of a novel chaotic system. Mathematical and ComputerModelling, 55 (2012), 1904-1915.

59. I. Pehlivan, I.M. Moroz and S. Vaidyanathan, Analysis, Synchronizationand Circuit Design of a Novel Butterfly Attractor. J. Of Sound And Vibration, 333 (2014) 5077-5096.

60. Ch K. Volos, I.M. Kyprianidis, and I.N. Stouboulos, Text encryption scheme realized with a chaotic pseudo-random bit generator, Journal of Engineering Science and Technology Review, vol. 6(4), pp. 9-14 (2013).

61. Ch.K. Volos, I.M. Kyprianidis, and I.N. Stouboulos, Image encryption process based on chaotic synchronization phenomena, Signal Processing, vol. 93(5), pp. 1328-1340 (2013).

62. D. Guégan, Chaos in economics and finance, Annual Reviews in Control, vol. 33, pp. 89-93 (2009).

63. Ch.K. Volos, I.M. Kyprianidis, and I.N. Stouboulos, Synchronization phenomena in coupled nonlinear systems applied in economic cycles, WSEAS Trans. Systems, vol. 11(12), pp. 681-690(2012).

64. P. Caraiani, Testing for nonlinearity and chaos in economic time series with noise titration, Economics Letters, vol. 120, pp. 192194 (2013)

65. A. $\mathrm{Wu}$ and $\mathrm{Z}$. Zeng, Anti-synchronization of a class of memristive recurrent neural networks, Communications in Nonlinear Science and Numerical Simulation, vol. 18, pp. 373385 (2013).

66. V. Sundarapandian, Output regulation of the Lorenz attractor, Far East Journal of Mathematical Sciences, vol. 42, pp. 289-299 (2010).

67. S. Vaidyanathan, Output regulation of Arneodo-Coullet chaotic system, Communications in Computer and Information Science, vol. 131, pp. 585-593 (2011).

68. S. Vaidyanathan, Output regulation of the unified chaotic system, Communications in Computer and Information Science, vol. 198, pp. 1-9 (2011).
69. S. Vaidyanathan, Output regulation of the Liu chaotic system, Applied Mechanics and Materials, vols. 110-116, pp. 3982-3989 (2012).

70. G. Chen, A simple adaptive feedback control method for chaos and hyper-chaos control, Applied Mathematics and Computation, vol. 217, pp. 7258-7264 (2011).

71. J. Zheng, A simple universal adaptive feedback controller for chaos and hyperchaos control, Computers \& Mathematics with Applications, vol. 61, pp. 2000-2004 (2011).

72. S. Vaidyanathan, Adaptive controller and synchronizer design for the Qi-Chen chaotic system, Lecture Notes of the Institute for Computer Sciences, Social-Informatics and Telecommunications Engineering, vol. 85, pp. 124-133 (2012).

73. V. Sundarapandian, Adaptive control and synchronization design for the Lu-Xiao chaotic system, Lecture Notes in Electrical Engineering, vol. 131, pp. 319-327 (2013).

74. S. Vaidyanathan, A ten-term novel 4-D hyperchaotic system with three quadratic nonlinearities and its control, International Journal of Control Theory and Applications, vol. 6, pp. 97-109 (2013).

75. S. Vaidyanathan, Analysis, control and synchronization of hyperchaotic Zhou system via adaptive control, Advances in Intelligent Systems and Computing, vol. 177, pp. 1-10 (2013).

76. D. Yang and J. Zhou, Connections among several chaos feedback control approaches and chaotic vibration control of mechanical systems, Communications in Nonlinear Science and Numerical Simulation, vol. 19, pp. 3954-3968 (2014).

77. M.T. Yassen, Chaos control of chaotic dynamical systems using backstepping design, Chaos, Solitons and Fractals, vol. 27, pp. 537-548 (2006).

78. J.A. Laoye, U.E. Vincent, and S.O. Kareem, Chaos control of 4D chaotic systems using recursive backstepping nonlinear controller, Chaos, Solitons and Fractals, vol. 39, pp. 356-362 (2009).

79. D. Lin, X. Wang, F. Nian, and Y. Zhang, Dynamic fuzzy neural networks modeling and adaptive backstepping tracking control of uncertain chaotic systems, Neurocomputing, vol. 73, pp. 2873-2881 (2010)

80. S. Vaidyanathan, Sliding mode control based global chaos control of Liu-Liu-Liu-Su chaotic system, International Journal of Control Theory and Applications, vol. 5, pp. 15-20 (2012).

81. S. Vaidyanathan, Global chaos control of hyperchaotic Liu system via sliding mode control, vol. 5, pp. 117-123 (2012).

82. L.M. Pecora and T.L. Carroll, Synchronization in chaotic systems, vol. 64, pp. 821-825 (1990).

83. S. Vaidyanathan and S. Rasappan, New results on the global chaos synchronization for Liu-Chen-Liu and Lü chaotic systems, Communications in Computer and Information Science, vol. 102, pp. 20-27 (2010).

84. S. Vaidyanathan and S. Rasappan, Hybrid synchronization of hyperchaotic Qi and Lü systems by nonlinear control, Communications in Computer and Information Science, vol. 131, pp. 585-593 (2011).

85. S. Vaidyanathan and K. Rajagopal, Anti-synchronization of Li and $\mathrm{T}$ chaotic systems by active nonlinear control, Communications in Computer and Information Science, vol. 198, pp. 175-184 (2011).

86. S. Vaidyanathan and S. Rasappan, Global chaos synchronization of hyperchaotic Bao and Xu systems by active nonlinear control, Communications in Computer and Information Science, vol. 198, pp. 10-17 (2011).

87. S. Vaidyanathan and K. Rajagopal, Global chaos synchronization of hyperchaotic Pang and Wang systems by active nonlinear control, Communications in Computer and Information Science, vol. 204, pp. 84-93 (2011).

88. S. Vaidyanathan, Hybrid chaos synchronization of Liu and Lü systems by active nonlinear control, Communications in Computer and Information Science, vol. 204, pp. 1-10 (2011).

89. P. Sarasu and V. Sundarapandian, Active controller design for generalized projective synchronization of four-scroll chaotic systems, International Journal of Systems Signal Control and Engineering Application, vol. 4, pp. 26-33 (2011).

90. S. Vaidyanathan and K. Rajagopal, Hybrid synchronization of hyperchaotic Wang-Chen and hyperchaotic Lorenz systems by active non-linear control, International Journal of Systems Signal Control and Engineering Application, vol. 4, pp. 55-61 (2011). 
91. S. Pakiriswamy and S. Vaidyanathan, Generalized projective synchronization of three-scroll chaotic systems via active control, Lecture Notes of the Institute for Computer Sciences, Social-Informatics and Telecommunications Engineering, vol. 85, pp. 146-155 (2012).

92. V. Sundarapandian and R. Karthikeyan, Hybrid synchronization of hyperchaotic Lorenz and hyperchaotic Chen systems via active control, Journal of Engineering and Applied Sciences, vol. 7, pp. 254-264 (2012).

93. R. Karthikeyan and V. Sundarapandian, Hybrid chaos synchronization of four-scroll systems via active control, Journal of Electrical Engineering, vol. 65, pp. 97-103 (2014).

94. E.M. Shahverdiev and K.A. Shore, Impact of modulated multiple optical feedback time delays on laser diode chaos synchronization, Optics Communications, vol. 282, pp. 35683572 (2009).

95. T. Botmart, P. Niamsup, and X. Liu, Synchronization of nonautonomous chaotic systems with time-varying delay via delayed feedback control, Communications in Nonlinear Science and Numerical Simulation, vol. 17, pp. 1894-1907 (2012).

96. S. Bowong, Adaptive synchronization between two different chaotic dynamical systems, Communications in Nonlinear Science and Numerical Simulation, vol. 12, pp. 976-985 (2007).

97. W. Lin, Adaptive chaos control and synchronization in only locally Lipschitz systems, Physics Letters A, vol. 372, pp. 31953200 (2008).

98. H. Salarieh and A. Alasty, Adaptive chaos synchronization in Chua's systems with noisy parameters, Mathematics and Computers in Simulation, vol. 79, pp. 233-241 (2008).

99. H. Salarieh and A. Alasty, Adaptive synchronization of two chaotic systems with stochastic unknown parameters, Communications in Nonlinear Science and Numerical Simulation, vol. 14, pp. 508-519 (2009).

100. S. Vaidyanathan and K. Rajagopal, Global chaos synchronization of Lü and Pan systems by adaptive nonlinear control, Communications in Computer and Information Science, vol. 205, pp. 193-202 (2011).

101. V. Sundarapandian and R. Karthikeyan, Anti-synchronization of Lü and Pan chaotic systems by adaptive nonlinear control, European Journal of Scientific Research, vol. 64, pp. 94-106 (2011).

102. V. Sundarapandian and R. Karthikeyan, Anti-synchronization of hyperchaotic Lorenz and hyperchaotic Chen systems by adaptive control, International Journal of Systems Signal Control and Engineering Application, vol. 4, pp. 18-25 (2011).

103. V. Sundarapandian and R. Karthikeyan, Adaptive antisynchronization of uncertain Tigan and Li systems, Journal of Engineering and Applied Sciences, vol. 7, pp. 45-52 (2012).

104. P. Sarasu and V. Sundarapandian, Generalized projective synchronization of three-scroll chaotic systems via adaptive control, European Journal of Scientific Research, vol. 72, pp. 504-522 (2012).

105. P. Sarasu and V. Sundarapandian, Generalized projective synchronization of two-scroll systems via adaptive control, International Journal of Soft Computing, vol. 7, pp. 146-156 (2012).

106. P. Sarasu and V. Sundarapandian, Adaptive controller design for the generalized projective synchronization of 4-scroll systems, International Journal of Systems Signal Control and Engineering Application, vol. 5, pp. 21-30 (2012).

107. S. Vaidyanathan and K. Rajagopal, Global chaos synchronization of hyperchaotic Pang and hyperchaotic Wang systems via adaptive control, International Journal of Soft Computing, vol. 7, pp. 28-37 (2012).

108. S.H. Lee, V. Kapila, M. Porfiri, and A. Panda, Master-slave synchronization of continuously and intermittently coupled sampled-data chaotic oscillators, Communications in Nonlinear
Science and Numerical Simulation, vol. 15, pp. 4100-4113 (2010).

109. X.Z. Jin and J.H. Park, Adaptive synchronization for a class of faulty and sampling coupled networks with its circuit implement, Journal of the Franklin Institute, vol. 351, pp. $4317-4333$ (2014).

110. C.K. Zhang, L. Jiang, Y. He, Q.H. Wu and M. Wu, Asymptotical synchronization for chaotic Lur'e systems using sampled-data control, Communications in Nonlinear Science and Numerical Simulation, vol. 18, pp. 2743-2751 (2013).

111. X. Xiao, L. Zhou, and Z. Zhang, Synchronization of chaotic Lur'e systems with quantized sampled-data controller, Communications in Nonlinear Science and Numerical Simulation, vol. 19, pp. 2039-2047 (2014).

112. S. Rasappan and S. Vaidyanathan, Global chaos synchronization of WINDMI and Coullet chaotic systems by backstepping control, Far East Journal of Mathematical Sciences, vol. 67, pp. 265-287 (2012).

113. S. Rasappan and S. Vaidyanathan, Synchronization of hyperchaotic Liu system via backstepping control with recursive feedback, Communications in Computer and Information Science, vol. 305, pp. 212-221 (2012).

114. S. Rasappan and S. Vaidyanathan, Hybrid synchronization of nscroll Chua and Lur'e chaotic systems via backstepping control with novel feedback, Archives of Control Sciences, vol. 22, pp. 343-365 (2012).

115. R. Suresh and V. Sundarapandian, Global chaos synchronization of a family of n-scroll hyperchaotic Chua circuits using backstepping control with recursive feedback, Far East Journal of Mathematical Sciences, vol. 73, pp. 73-95 (2013).

116. S. Rasappan and S. Vaidyanathan, Hybrid synchronization of n-scroll chaotic Chua circuits using adaptive backstepping control design with recursive feedback, Malaysian Journal of Mathematical Sciences, vol. 7, pp. 219-246 (2013).

117. S. Vaidyanathan and S. Rasappan, Global chaos synchronization of n-scroll Chua circuit and Lur'e system using backstepping control design with recursive feedback, Arabian Journal for Science and Engineering, vol. 39, pp. 3351-3364 (2014).

118. S. Rasappan and S. Vaidyanathan, Global chaos synchronization of WINDMI and Coullet chaotic systems using adaptive backstepping control design, Kyungpook Mathematical Journal, vol. 54, pp. 293-320 (2014).

119. H.T. Yau, Chaos synchronization of two uncertain chaotic nonlinear gyros using fuzzy sliding mode control, Mechanical Systems and Signal Processing, vol. 22, pp. 408-418 (2008).

120. H. Li, X. Liao, C. Li, and C. Li, Chaos control and synchronization via a novel chatter free sliding mode control strategy, Neurocomputing, vol. 74, pp. 3212-3222 (2012).

121. S. Vaidyanathan and S. Sampath, Global chaos synchronization of hyperchaotic Lorenz systems by sliding mode control, Communications in Computer and Information Science, vol. 205, pp. 156-164 (2011).

122. V. Sundarapandian and S. Sivaperumal, Sliding controller design of hybrid synchronization of four-wing chaotic systems, International Journal of Soft Computing, vol. 6, pp. 224-231 (2011).

123. S. Vaidyanathan and S. Sampath, Anti-synchronization of fourwing chaotic systems via sliding mode control, International Journal of Automation and Computing, vol. 9, pp. 274-279 (2012).

124. S. Vaidyanathan, Global chaos synchronisation of identical Li$\mathrm{Wu}$ chaotic systems via sliding mode control, International Journal of Modelling, Identification and Control, vol. 22, no. 2, pp. 170-177 (2014).

125. H.K. Khalil, Nonlinear System, $3^{\text {rd }}$ ed.,Prentice Hall,New Jersey, USA (2002). 